\title{
The importance of Helicobacter pylori in the development of gastric MALT lymphoma - induction of proliferation and immune suppression
}

\author{
Paweł Krzyżek¹, Barbara Kwiatkowska²
}

Marginal zone B-cell lymphomas are indolent, slow-growing lymphomas derived from mature B cells. They represent about $8 \%$ of all lymphomas and about $50 \%$ of all primary gastric lymphomas. Based on numerous epidemiological and microbiological studies, Helicobacter pylori is believed to be responsible for the progression of gastric MALT lymphomas (GML). Lymphoid tissue is physiologically absent from the stomach. However, GML can arise from chronic H. pylori infection and immune cell infiltration. This review article describes the mechanisms favouring the development of $H$. pylori-induced GML, and suggests potential targets for a more effective remission of lymphomas localized within the stomach.

NOWOTWORY J Oncol 2017; 67, 4: 261-266

Key words: Helicobacter pylori, immunosuppression, MALT lymphoma, proliferation, stomach

\section{Introduction}

Helicobacter pylori is a spiral and gram-negative bacterium capable of colonizing the gastric mucosa. This process occurs most often during early childhood and lasts throughout life in the absence of any adequate eradication therapy [1]. Not all people colonized with this bacteria exhibit disease symptoms, but importantly this condition predisposes towards their development [2]. It is estimated that over $90 \%$ of chronic gastritis is a result of prolonged $H$. pylori infection. Other causes include non-steroidal anti-inflammatory drugs, alcohol consumption, gastroesophageal reflux disease and autoimmune mucosa atrophy. Patients with gastritis suffer from gastrointestinal disorders which include dyspepsia, bloating, discomfort and feeling of fullness in the upper abdomen. Symptoms may be aggravated by unhealthy diets, alcohol consumption and emotional stress or fatigue [3]. The acute phase of $H$. pylori infection is associated with an intense inflammation of the gastric mucosa, characterized by infiltration of immune system cells; especially neutrophils. Increased production of proinflammatory cytokines involved in gastric tissue degradation, (including IL-1 $\beta$, IL-6, IL-8, IL-12, TNF- $\alpha$, CCL2-5, CCL20 and CXCL1-3), is the consequence [4]. Inflammatory mediators cause the following: epithelial lesion formation, changes in proliferation and apoptosis processes along with modulating host gene expression [2]. The acute phase is followed by a decreased antimicrobial response of the host organism and chronic H. pylori infection. Some people may develop gastric and duodenal ulcers, gastric cancer or mucosal associated lymphoid tissue (MALT) lymphoma [1].

\section{Characteristics of the MALT lymphoma}

MALT lymphomas can affect the majority of human body organs including lungs, thyroid gland, breast, urinary bladder and skin [5]. MALT lymphomas are most commonly found in the digestive system, particularly the stomach, which are defined as gastric MALT lymphomas (GML) [6]. GML is an indolent extranodal marginal zone B cell lymphoma (MZL). The growth of primary gastrointestinal tract lymphomas, including gastric lymphoma, is generally slow and limited to the gastrointestinal wall; it may remain asymptomatic for many years. The morbidity rates of GML increases with age. GML are diagnosed mainly in patients aged 60 years or over, and GML rates are almost the same for men and

\footnotetext{
${ }^{1}$ Department of Microbiology, Wroclaw Medical University, Poland

${ }^{2}$ Department of Internal and Occupational Diseases, Hypertension and Clinical Oncology,

Wroclaw Medical University, Poland
} 
women [7]. MZL are non-Hodgkin lymphomas which evolve from mature B cells [8]. Lymphoid tissue is physiologically absent from the stomach. However, due to chronic H. pylori infection and an infiltration of immune cells, gastric MALT lymphomas can arise [9]. MALT lymphoma cells share the same cytological and immunophenotypical features: i.e. $\mathrm{CD}_{20}{ }^{+}, \mathrm{CD}_{21}{ }^{+}, \mathrm{CD}^{2} 5^{+}, \operatorname{lgM}^{+}$and lgD- [10]. MALT lymphomas are classified as rare diseases. The annual estimated incidence of GML is approximately $1-1.5$ per 100000 . In comparison, gastric cancer is 5 to 10 times more common (United States National Cancer Institute Surveillance Epidemiology and End Results data) [11].

The primary GML diagnosis should be based on clinical symptoms and on immunohistochemical and pathohistological examination of gastric biopsy tissue [11, 12]. Before undertaking such examinations, it is important to interview the patient, focusing on clinical symptoms that typically include: dyspepsia, epigastric pain, nausea, vomiting, weight loss and any alarming symptoms, such as melena and hematemesis. Some patients however can remain asymptomatic. The gold standard of the GML diagnostic process is an endoscopic examination with multiple biopsies of gastric tissue $[7,8]$. Macroscopic changes defined by gastroscopy are nonspecific including thickening of mucosal folds and irregular masses with superficial erosions and superficial ulcers. Due an equivocal endoscopic picture, multiple biopsies are obligatory for pathohistological examination. Sometimes immunophenotyping and molecular examinations may be necessary [8, 12].

To evaluate the staging of both Hodgkin and non-Hodgkin lymphomas the Ann Arbor system is commonly used. However, due to its limitations for assessing primary gastrointestinal lymphomas, the Lugano and Radaszkiewicz classifications have been proposed [11]. In order to more precisely evaluate the staging of primary gastrointestinal lymphomas, the European Gastro-Intestinal Lymphoma Study Group created The Paris System; a special modification of the TNM (tumor-node-metastasis) staging system, used for classifying non-hematologic solid malignancies [13]. There are several genetic aberrations characteristic to lymphoma cells: translocations, point mutations, gene deletions and amplifications. Detecting their presence is crucial in the diagnostic process and affects the treatment of choice [11]. The genetic aberrations at translocations $t(1 ; 14)(p 22 ; q 32)$ and $t(11 ; 18)(q 21 ; q 21)$ are responsible for the response to apoptosis signals being ineffective and increased transcriptional activity of NF- $\kappa \mathrm{B}$ (nuclear factor kappa-light-chain-enhancer of activated $B$ cells). Other detected translocations, $t(14 ; 18)(q 32 ; q 21)$ and $t(3 ; 14)$ (p14;q32), are rare and their significance has not yet been defined precisely [12]. The most frequent chromosomal aberration is the $t(11 ; 18)$ translocation which is detected in approximately $20 \%$ of patients with MALT Iymphoma, particularly those with MALT lymphomas located in the stomach, colon and lung [5].

\section{Expression of $H$. pylori virulence factors and GML development}

Based on numerous epidemiological and microbiological studies $H$. pylori is believed to be responsible for GML progression $[5,6]$. In a study on 110 patients with GML, H. pylori infection was detected in 101 (92\%) cases [14]. It is believed that $H$. pylori strains producing multiple virulence factors are associated with exacerbating gastritis. Correlations between the expression of virulence factors, especially CagA oncoprotein and VacA toxin, are relatively well documented in peptic ulcers and gastric cancer. Nevertheless, their involvement in the development of gastric MALT lymphomas remains controversial [6].

The cagPAl pathogenic island is a $40 \mathrm{~kb}$ DNA fragment containing 27 to 31 genes; eighteen of which encode information for proteins forming the IV secretory system (T4SS), which facilitates CagA oncoprotein and peptidoglycan fragment translocation [2]. CagA is a 120-145 kDa protein produced by $60-70 \%$ of $H$. pylori strains. It rearranges the host actin cytoskeleton, disrupts tight junctions and causes morphological changes of eukaryotic cells leading to the so-called hummingbird phenotype [4]. Moreover, CagA inhibits the apoptosis regulator and tumor suppressor - p53, and thus allows B cells to avoid programmed death as well as in mediating the accumulation of genetic mutations [15].

There are two pathways by which CagA deregulates intracellular signalling pathways in eukaryotic cells; i.e. phosphorylation-dependent and-independent. The first is based on tyrosine phosphorylation within the EPIYA (Glu-Pro-IleTyr-Ala) motifs of the CagA protein. Modified protein induces tyrosine phosphatase 2 (SHP-2) and this activates extracellular signal-regulated kinases $1 / 2$ (Erk1/2). The unphosphorylated CagA protein induces Erk1/2 kinase through an alternative Ras-Raf signalling pathway $[2,16]$. Both processes contribute to activating the NF- $\kappa$ B transcription factor and increased IL-8 expression [17]. Moreover, peptydoglycan fragments delivered through T4SS to host cells also activate $\mathrm{NF}-\kappa \mathrm{B}$ and increase IL- 8 secretion by inducing Nod 1 proteins (nucleotide-binding oligomerization domain 1) [18]. IL-8 is not the only chemoattractant for leucocytes but also plays an important role as a mitogenic and angiogenic factor in neoplasm progression [19]. Erk1/2 stimulation and p53 expression blockade facilitates apoptosis inhibition which results in genetically dysfunctional cells being produced. This may disrupt homeostasis between programmed cell death and proliferative processes together with H. pylori-mediated gastric MALT lymphomas development [16]. Ohnishi et al. engineered transgenic mice that endogenously produce CagA proteins. After 72 weeks from transfection, gastric 
epithelial hyperplasia was observed and in some individuals polyps and adenocarcinomas were detected. Such changes have been shown to be due to hyperactivity of SHP-2 phosphatase and Erk1/2 kinase [20].

Many epidemiological studies have attempted to elucidate any correlation between CagA production by H. pylori and the presence of gastric MALT lymphomas. When studying CagA seroprevalence in H. pylori infected patients, Eck et al. obtained positive outcomes in 95.5\% (64/67) GML patients and $67 \%$ (33/49) in controls with chronic gastritis [21]. Sumida et al. demonstrated an identical prevalence of anti-CagA antibodies, i.e. 95.5\% (42/44). Furthermore, it was found that patients in GML remission (86.4\%; 38/44) had significantly higher levels of anti-H. pylori and anti-CagA antibodies than in the group without the therapeutic effect; respectively $105.2 \pm 166.4$ vs $24.3 \pm 14.9 \mathrm{U} / \mathrm{mL}$ and 43.7 \pm 25.1 vs $16.5 \pm 13.2 \mathrm{U} / \mathrm{mL}$ [22]. A study by Delchier et al. divided patients into two groups, based on disease progression, i.e. to low-grade GML (LGML) and diffuse large B-cell lymphoma (DLBCL). In the first group, antibodies against CagA were detected in $44.8 \%$ (13/29) of patients, while a higher seroprevalence was found in the DLBCL group of 75\% (12/16) [23]. Similar observations were made by Peng et al., who identified cagA through isolating DNA from patients' biopsies where gene prevalence was demonstrated in $30.3 \%(17 / 56)$ of patients with gastritis, $37.8 \%(14 / 37)$ with LGML and 76.7\% (23/30) with DLBCL [24]. These results suggest that the gastric MALT lymphoma progression may be dependent on the expression of cagA by H. pylori strains, which is indirectly confirmed by GML treatment effects in $\mathrm{Hp}^{+} \mathrm{CagA}^{+}$patients. Disease remission after eradicating $H$. pylori was found to be faster in patients with $\mathrm{CagA}^{+} \mathrm{H}$. pylori infection (average 3 months) than in patients infected with CagA- bacterial strains (6.5 months) [25].

The second most important virulence factor produced by H. pylori is VacA. All strains of this bacterium have a vacA gene but in vitro only half of them synthesize this toxin [6]. Three variable regions are present in the vacA sequence, i.e. the signal sequence $s(s 1 a, s 1 b, s 1 c, s 2)$, the middle region $m(m 1, m 2 a, m 2 b)$ and the intermediate region $i(i 1, i 2, i 3)$. The $\mathrm{s} 1 / \mathrm{m} 1 / \mathrm{i} 1$ variants have higher biological activity and a stronger toxic effect [26]. VacA is a highly immunogenic $95 \mathrm{kDa}$ protein causing a number of pathological changes in the targeted host cells. Among these, is its ability to form anion-selective pores in cell membranes, interfere with cytoskeleton-dependent cell function and induce apoptosis via the mitochondrial cytochrome $c$ release $[2,4]$. In addition, VacA has in vivo antiproliferative activity against lymphocytes and the ability of modulating immune system processes [6]. Koehler et al. isolated DNA from biopsy samples of $H$. pylori infected patients with gastritis, gastric cancer and GML. It was estimated that in gastric cancer patients the dominant $H$. pylori genotype was vacAs $1 \mathrm{~m} 1$ (s1 alleles were detected in $93 \%, 26 / 28$, and $\mathrm{m} 1$ in $61 \%$, $17 / 28$ ) whilst in the GML group the vacAs $1 \mathrm{~m} 2$ variant was dominant (alleles were found in $83 \%, 20 / 24$, and $83 \%$ in 20/24) [27]. The middle region affects the toxin's ability to interact with targeted cells [26]. Thus the vacAm2 variant reduces antiproliferative activity [6]. For this reason, it is proposed that the presence of less active VacA toxin forms, produced by $H$. pylori strains, may contribute towards gastric MALT lymphomas developing.

\section{Immune response modulation by $H$. pylori}

Chronic inflammation of the gastric mucosa caused by H. pylori infection is probably the key factor responsible for GML development. The inflamed gastric mucosa furthers increased antigen presentation and recruitment of B cells [28]. Infiltration of these immune cells is accompanied by activated proliferative processes and the formation of B cell clusters and germinal centers (GC) $[12,16]$. Moreover, abnormal $\mathrm{NF}-\kappa \mathrm{B}$ pathway activation initiates the transformation of gastric lymphoid tissue [12]. The transcription factor NF- $\kappa \mathrm{B}$ controls $B$ and $T$ cells maturation and survival, exerting an anti-apoptotic effect on these cells [29]. Through CagA and peptidoglycan fragment translocation, $H$. pylori increases NF- $\kappa$ B activity which results in the host immune response being modulated, hyperproliferation of lymphocytes and the potential induction of GML $[2,17,18]$. Thereby, chronic or recurrent activation of the immune system by $\mathrm{H}$. pylori can lead to lymphoid tissue growth. As supported by coexisting environmental factors and genetic predispositions this process can lead to hyperproliferation of lymphoid tissue and oncogenesis $[2,8]$. Despite the possibility of $B$ cells to undergo autostimulation-dependent activation by H. pylori antigens, lymphogenesis may also be mediated by T-cell stimulation $[6,12]$.

The main part of the host lymphocytes induced by H. pylori are the $T h_{1}$ and $T h_{17}$ populations. The first of these secrete proinflammatory cytokines IFN- $\gamma$ and TNF- $\alpha$, which in turn activate antimicrobial activity in macrophages. $\mathrm{Th}_{17}$ cells also affect progression of the inflammation process through secretion of inflammatory mediators such as IL-17A, IL-17F, IL-21 and IL-22 [4]. This process is characteristic of the initial stages of $H$. pylori infection. The next step is based on the host immune response being suppressed by over-activation of $\mathrm{CD} 4^{+} \mathrm{CD} 25^{+}$(Treg cells) $[9,30,31]$. Treg cells recruitment occurs through secretion of CCL17 and CCL22 chemokines produced by B cells [32]. Treg cells promote tolerogenic signals and secrete anti-inflammatory cytokines, such as IL-10 and TGF- $\beta[9,30]$. IL-10 increases the $B$ cells ability to proliferate, survive and differentiate. On the other hand, these mediators also have antiproliferative and suppressive effects on other fractions of immune cells, i.e. they reduce secretion of proinflammatory IFN- $\gamma$ and TNF- $\alpha$ by Th $h_{1}$ cells and of IL- 17 by Th ${ }_{17}$ cells. Such ac- 
tivities promote carcinogenesis via inhibiting secretion of antitumor mediators [33]. TGF- $\beta$ is a cytokine that inhibits excessive proliferation and repressor genes encoding for cytotoxic mediators, including perforin, granzymes $\mathrm{A}$ and $\mathrm{B}$ and IFN- $\gamma$ [34]. The suppressive environment mediated by high TGF- $\beta$ concentrations affords protection against tumours developing. This happens until carcinogenic cells become resistant to the stimuli initiated by this mediator, for example by the increased activity of mammalian target of rapamycin (mTOR), which makes carcinogenic cells insensitive to TGF- $\beta$-mediated antiproliferative activity [35]. In such situations, elevated TGF- $\beta$ level is beneficial for lymphoma development because it disrupts cytotoxic and proapoptotic activity to tumor-altered cells [33]. In an in vivo mouse model, Craig et al. demonstrated the ability of Helicobacter spp. to promote MALT lymphoma development by Treg cells activation. In H. felis infected BALB/c mice, depletion of $\mathrm{CD}^{+}$or $\mathrm{CD} 25^{+}$by monoclonal antibodies resulted in tumor regression. After 18 months post-infection, 2-15 tumors were found in each individual mouse stomach. A different situation was observed in mice lacking $C D 4^{+} \mathrm{T}$ cells because none had developed lymphoma. In mice without $\mathrm{CD} 25^{+} \mathrm{T}$ cells studies show similar results, excepting one individual with a single tumor [32]. Such results suggest that Treg cells can actively affect oncogenesis development via promoting a suppressive environment that reduces antitumor immune system activity.

In addition to the ability for recruiting Treg cells by $H$. pylori, this microorganism may suppress the excessive proinflammatory response by promoting high $\mathrm{B} 7 / \mathrm{H} 1$ levels. These molecules are homologous to immunoglobulins and exert an inhibitory effect on programmed death-1 (PD-1) receptors on the T cells' surface. Experiments on tumor cell lines have shown that $\mathrm{B} 7 / \mathrm{H} 1$ facilitates carcinogenic resistance to apoptosis mediated by Fas-induced cell-death. What's more, $H$. pylori increases $B 7 / \mathrm{H} 1$ expression which is also responsible for the transformation of virgin T cells into Treg cells [37]. A study by Lina et al. showed that H. pylori increases $\mathrm{B} 7 / \mathrm{H} 1$ concentrations which was dependent on $\mathrm{CagA}$ and peptidoglycan fragment translocation into host cells. The in vivo effect of two H. pylori strains on B7/ $/ \mathrm{H} 1$ expression during $\mathrm{C} 57 \mathrm{BL} / 6$ mice infection was verified, i.e. PMSS1 - producing the T4SS system, and SS1 - having no functional T4SS. Enhancement of increased B7/H1 concentrations was observed only for the H. pylori PPSS1 strain, which in turn resulted in elevated bacterial growth in the mouse and higher amounts of Treg cells and raised serum IL-10 [38].

\section{Gastric MALT lymphoma treatment}

MALT lymphomas are distinguished by an indolent clinical course. Most are diagnosed at an early stage. Therapeutical choices depend on whether $H$. pylori infection and the presence of $(t 11 ; 18)$ translocation are confirmed; the latter being reported as an adverse prognostic factor in terms of lymphoma regression after $H$. pylori eradication [8]. Chronic H. pylori infection can lead to lymphoid proliferation and GML development. For this reason, H. pylori infection should be confirmed in each patient diagnosed with GML. The diagnosis should be based on invasive and noninvasive tests including measuring $\lg G, \lg M$, IgA antibodies, monoclonal stool antigens detection, the ${ }^{13} \mathrm{C}$-urea breath test, microbes culture and histochemical testing [39]. H. pylori eradication therapy should rely on highly effective antibiotics with due consideration given to possible resistance in bacterial strains [5]. Approximately $20 \%$ of $H$. pylori strains are proved to be clarithromycin resistant. As a consequence, it is strongly recommended to attempt an eradication by quadruple therapy using a bismuth salt, tetracycline, metronidazole and PPI instead of triple therapy using PPI, clarithromycin and amoxicillin [40]. H. pylori antibiotic therapy can lead to complete GML remission in $77.5-94 \%$ of patients [16]. Gastric lymphoma regression in response to $H$. pylori eradication can on average take from 5 to 12 months and sometimes even 45 months. After achieving remission, systematic clinical and endoscopic monitoring is mandatory including pathohistological examinations and tests for H. pylori infection [7]. The estimated survival rate of patients with complete lymphoma remission can reach to almost $100 \%$ after 10 years. Patients with partial remission have a 10 -year survival rate of $80 \%[7,41]$. H. pylori reinfection determines MALT lymphoma recurrence. In such cases, disease progression is more dynamic as a consequence of gastric tissue sensitivity to $H$. pylori antigens [42]. In cases when pharmacological treatment fails, radiotherapy should be attempted. In patients with aggressive primary $G M L$, i.e. large B-cell lymphoma (DLBCL), systemic polychemotherapy is recommended for first-line treatment despite the coexisting $H$. pylori infection. Patients therapy whenever advanced-stage disease occurs and in those with confirmed lymphoma recurrence is based on chlorambucil, fludarabine and rituximab treatment $[5,7,9,41]$.

\section{Implications for medical practice and teaching}

Confirming $H$. pylori status in people with diagnosed GML is a routine procedure [39]. It is suggested that besides detecting such bacteria, an important diagnostic element may also be in determining the $H$. pylori virulence profile. Such practice is important not only for epidemiological reasons (e.g. higher isolation frequency of VacAs $1 \mathrm{~m} 2$ strains), but also in planning an effective therapy. It was found that GML remission caused by $\mathrm{CagA}^{+} H$. pylori strains is twice as fast as that of $\mathrm{CagA}^{-}$strains [25]. Henceforth, the presence of the cagA gene may in future be treated as a microbial genetic marker which defines the duration of patient convalescence. 
Moreover when toxins production by H. pylori becomes known then appropriate compounds can be incorporated that inhibit pathological metabolic changes in those eukaryotic cells exposed to bacterial toxins. Oncoprotein CagA induces rearrangement of the host actin cytoskeleton, disrupts tight junctions, elicits morphological changes and causes malignant transformation [4]. Inhibition of CagA kinases in $\mathrm{H}$. pylori infected cells leads to a complete loss of CagA phosphorylation and decreases cell death. Pharmacological inhibition of tyrosine kinases, which mediate CagA phosphorylation, may thus become an attractive treatment option for patients with late stage GML or in those where H. pylori eradication proves unsuccessful [43]. Key molecules responsible for cell-cycle regulation, proliferation and apoptosis that are present in the process of MALT lymphoma oncogenesis may become specific therapy targets not only in patients with this kind of lymphoma, but also in patients with other lymphoproliferative disorders [11].

Increased levels of Treg cell recruitment and infiltration within tumor-altered stomach tissue is observed [32]. Thus in the course of GML, the inflammation intensity correlates often inversely with the density of $H$. pylori found in the stomach. On this basis, it can be falsely concluded that the absence of a strong inflammatory reaction in the gastric mucosa may suggest a low-grade disease. Finding a high bacterial titre with low-grade mucosal inflammation may, however, indicate the development of the GML process [44]. For this reason, attention should be paid to an increased oncological vigilance in physicians diagnosing patients whenever gastric lymphomas are suspected.

Elevated Treg cells level in the gastric mucosa is a key factor in reducing proliferation and cytotoxic activity of other T cells subpopulations, therapeutic failure and a poor prognosis [9, 30,45,46]. Studies on Treg cell activity during an ongoing $H$. pylori infection may help to find new and effective therapies focused on oncogenesis control. The course of tumor immunotherapy has shown that Treg-targeting compounds may be good candidates for treatment based on immune system modulation. Reducing Treg activity promotes the shift of the tumor microenvironment from being strongly suppressive to the physiologically active $[45,46]$. Among potential methods that can achieve this effect are: IL-10 receptor blockade [47], selectin blockade [48], inhibition of chemokines responsible for Treg cells recruitment (CCL17, CCL22, CCL20 or CXCL13), blocking chemokine receptors specifically expressed by Treg effector cells (e.g. CCR4), inhibition of CTLR-4 co-inhibitory molecules expressed constitutively on the surface of Treg cells, or exogenous IL-2 administration (capable of reducing suppressive activity of Treg cells) [46]. In addition to this Treg cell-blocking therapy, it may also prove effective to administer low doses of cyclophosphamide. Such therapy selectively reduces the amount of rapidly proliferating Treg cells within tumor tissue and strengthens the anti-tumor response [49].

\section{Conclusions}

Numerous studies report a strong association between H. pylori gastric mucosa colonization and GML development. H. pylori infection mediates Treg cell recruitment, which in turn promotes tolerogenic signals and the secretion of anti-inflammatory cytokines IL-10 and TGF- $\beta$. In recent years, an increasing number of reports suggest a key role of Treg cells in perturbing cytotoxic and proapoptotic activity and probably an effect on GML development. Therefore, decrease of the activity or over-recruitment of these immune cells appears to be an important target in anti-tumor therapies based on enhancing the sensitivity of neoplastic cells. The complexity of GML pathogenesis requires extensive knowledge of oncology, microbiology and immunology to ensure delivery of effective treatment to the patient. In the light of the presented conclusions, close collaboration between gastroenterologists, oncologists and microbiologists is thus proposed as a standard procedure in the battle against GML.

\section{Conflict of interest: none declared}

\section{Paweł Krzyżek, MD}

Department of Microbiology

Wroclaw Medical University

4 Chałubińskiego St.

50-368 Wrocław, Poland

e-mail:krojcerpawel@gmail.com

Received: 2 May 2017

Accepted: $10 \mathrm{Jul} 2017$

\section{References}

1. Blaser MJ, Atherton JC. Helicobacter pylori persistence: biology and disease. J Clin Invest 2004; 113: 321-333.

2. Subhash VV, Ho B. Inflammation and proliferation - a causal event of host response to Helicobacter pylori infection. Microbiology 2015; 161: 1150-1160.

3. Genta RM, Sonnenberg A. Helicobacter-negative gastritis: a distinct entity unrelated to Helicobacter pylori infection. Aliment Pharmacol Ther 2015; 41: 218-226.

4. White JA, Winter JR, Robinson K. Differential inflammatory response to Helicobacter pylori infection: etiology and clinical outcomes. J Inflamm Res 2015; 8: 137-147

5. Witkowska M, Smolewski P. Helicobacter pylori infection, chronic in flammation, and genomic transformations in gastric MALT lymphoma. Mediators Inflamm 2013; 2013: 523170.

6. Floch P, Mégraud F, Lehours P. Helicobacter pylori strains and gastric MALT lymphoma. Toxins 2017; 9: E132.

7. Park JB, Koo JS. Helicobacter pylori infection in gastric mucosa-associated lymphoid tissue lymphoma. World J Gastroenterol 2014; 20: 2751-2759.

8. Hu Q, Zhang Y, Zhang X et al. Gastric mucosa-associated lymphoid tissue lymphoma and Helicobacter pylori infection: a review of current diagnosis and management. Biomark Res 2016; 4: 15.

9. Kuo SH, Cheng AL. Helicobacter pylori and mucosa-associated lymphoid tissue: what's new. Hematology 2013; 2013: 109-117.

10. Troppan K, Wenzl K, Neumeister P et al. Molecular pathogenesis of MALT lymphoma. Gastroenterol Res Pract 2015; 2015: 102656.

11. Pereira MI, Medeiros JA. Role of Helicobacter pylori in gastric mucosa-associated lymphoid tissue lymphomas. World J Gastroenterol 2014; 20: 684-698. 
12. Owens SR, Smith LB. Molecular aspects of H. pylori-related MALT lymphoma. Patholog Res Int 2011; 2011: 193149.

13. Ruskoné-Fourmestraux A, Dragosics B, Morgner A et al. Paris staging system for primary gastrointestinal lymphomas. Gut 2003; 52: 912-913.

14. Wotherspoon AC, Ortiz-Hidalgo C, Falzon MR et al. Helicobacter pylori-associated gastritis and primary B-cell gastric lymphoma. Lancet 1991; 338: 1175-1176.

15. Umehara $\mathrm{S}$, Higashi $\mathrm{H}$, Ohnishi $\mathrm{N}$ et al. Effects of Helicobacter pylori CagA protein on the growth and survival of B lymphocytes, the origin of MALT lymphoma. Oncogene 2003; 22: 8337-8342.

16. Wang HP, Zhu YL, Shao W. Role of Helicobacter pylori virulence factor cytotoxin-associated gene $A$ in gastric mucosa-associated lymphoid tissue lymphoma. World J Gastroenterol 2013; 19: 8219-8226.

17. Nozawa Y, Nishihara K, Peek RM et al. Identification of a signaling cascade for interleukin-8 production by Helicobacter pylori in human gastric epithelial cells. Biochem Pharmacol 2002; 64: 21-30.

18. Viala J, Chaput C, Boneca IG et al. Nod1 responds to peptidoglycan delivered by the Helicobacter pylori cag pathogenicity island. Nat Immunol 2004; 5: 1166-1174.

19. Xie K. Interleukin-8 and human cancer biology. Cytokine Growth Factor Rev 2001; 12: 375-391.

20. Ohnishi N, Yuasa H, Tanaka S et al. Transgenic expression of Helicobacter pylori CagA induces gastrointestinal and hematopoietic neoplasms in mouse. Proc Natl Acad Sci USA 2008; 105: 1003-1008.

21. Eck M, Schmausser B, Haas R et al. MALT-type lymphoma of the stomach is associated with Helicobacter pylori strains expressing the CagA protein. Gastroenterology 1997; 112: 1482-1486.

22. Sumida T, Kitadai Y, Hiyama T et al. Antibodies to Helicobacter pylori and CagA protein are associated with the response to antibacterial therapy in patients with $\mathrm{H}$. pylori-positive API2-MALT1-negative gastric MALT lymphoma. Cancer Sci 2009; 100: 1075-1081.

23. Delchier JC, Lamarque $D$, Levy $M$ et al. Helicobacter pylori and gastric lymphoma: high seroprevalence of CagA in diffuse large B-cell lymphoma but not in low-grade lymphoma of mucosa-associated lymphoid tissue type. Am J Gastroenterol 2001; 96: 2324-2328.

24. Peng H, Ranaldi R, Diss TC et al. High frequency of CagA+ Helicobacter pylori infection in high-grade gastric MALT B-cell lymphomas. J Pathol 1998; 185: 409-412.

25. Kuo SH, Chen LT, Lin CW et al. Detection of the Helicobacter pylori CagA protein in gastric mucosa-associated lymphoid tissue lymphoma cells: clinical and biological significance. Blood Cancer J 2013; 3: e125.

26. Jones KR, Jang S, Chang JY et al. Polymorphisms in the intermediate region of VacA impact Helicobacter pylori-induced disease development. J Clin Microbiol 2011; 49: 101-110.

27. Koehler $\mathrm{Cl}$, Mues MB, Dienes $\mathrm{HP}$ et al. Helicobacter pylori genotyping in gastric adenocarcinoma and MALT lymphoma by multiplex PCR analyses of paraffin wax embedded tissues. Mol Pathol 2003; 56: 36-42.

28. Suarez $\mathrm{F}$, Lortholary $\mathrm{O}$, Hermine $\mathrm{O}$ et al. Infection-associated lymphomas derived from marginal zone B cells: a model of antigen-driven lymphoproliferation. Blood 2006; 107: 3034-3044.

29. Gerondakis S, Siebenlist U. Roles of the NF-kappaB pathway in lymphocyte development and function. Cold Spring Harb Perspect Biol 2010; 2: a000182.

30. AiTL, Solomon BD, Hsieh CS. T-cell selection and intestinal homeostasis. Immunol Rev 2014; 259: 60-74.
31. Raghavan S, Suri-Payer E, Holmgren J. Antigen-specific in vitro suppression of murine Helicobacter pylori-reactive immunopathological T cells by CD4CD25 regulatory T cells. Scand JImmunol 2004; 60: 82-88.

32. Craig VJ, Cogliatti SB, Arnold I et al. B-cell receptor signaling and CD40 ligand-independent $T$ cell help cooperate in Helicobacter-induced MALT lymphomagenesis. Leukemia 2010; 24: 1186-1196.

33. Taylor JG, Gribben JG. Microenvironment abnormalities and lymphomagenesis: Immunological aspects. Semin Cancer Biol 2015; 34: 36-45.

34. Thomas DA, Massagué J. TGF- $\beta$ directly targets cytotoxic T cell functions during tumor evasion of immune surveillance. Cancer Cell 2005; 8: 369-380.

35. Sebestyén A, Márk Á, Hajdu M et al. Rapamycin can restore the negative regulatory function of transforming growth factor beta 1 in high grade lymphomas. Cytokine 2015; 73: 219-224.

36. Azuma T, Yao S, Zhu G et al. B7-H1 is a ubiquitous antiapoptotic receptor on cancer cells. Blood 2008; 111: 3635-3643.

37. Beswick EJ, Pinchuk IV, Das S et al. Expression of the programmed death ligand 1, B7-H1, on gastric epithelial cells after Helicobacter pylori exposure promotes development of CD4+CD25+ FoxP3+ regulatory T cells. Infect Immun 2007; 75: 4334-4341.

38. Lina TT, Alzahrani S, House J et al. Helicobacter pylori cag pathogenicity island's role in B7-H1 induction and immune evasion. PLoS One 2015 10: e0121841.

39. Patel SK, Pratap CB, Jain AK et al. Diagnosis of Helicobacter pylori: what should be the gold standard? World J Gastroenterol 2014; 20: 12847-12859.

40. Mégraud F. The challenge of Helicobacter pylori resistance to antibiotics: the comeback of bismuth-based quadruple therapy. Therap Adv Gastroenterol 2012; 5: 103-109.

41. Kalinka-Warzocha E. Optymalizacja leczenia chłoniaków B-komórkowych o niskim stopniu złośliwości. Onkol Prakt Klin 2007; 3: 140-149.

42. Cammarota G, Montalto M, Tursi A et al. Helicobacter pylori reinfection and rapid relapse of low-grade B-cell gastric lymphoma. Lancet 1995; 345: 192.

43. Krisch LM, Posselt G, Hammerl P et al. CagA phosphorylation in Helicobacter pylori-infected $B$ cells is mediated by the nonreceptor tyrosine kinases of the Src and Abl families. Infect Immun 2016; 84: 2671-2680.

44. Laur AM, Floch $\mathrm{P}, \mathrm{Chambonnier} L$ et al. Regulatory $T$ cells may participate in Helicobacter pylori persistence in gastric MALT lymphoma: Lessons from an animal model. Oncotarget 2016; 7: 3394-3402.

45. Romero-Adriàn TB, Leal-Montiel J. Helicobacter pylori infection: Regulatory $T$ cells and their participation in the immune response. Jundishapur JMicrobiol 2013; 6: e5183.

46. Tanaka A, Sakaguchi S. Regulatory T cells in cancer immunotherapy. Cell Res 2017; 27: 109-118.

47. Hart KM, Byrne KT, Molloy MJ et al. IL-10 immunomodulation of myeloid cells regulates a murine model of ovarian cancer. Front Immunol 2011; 2: 29.

48. Barthel SR, Gavino JD, Descheny L et al. Targeting selectins and selectin ligands in inflammation and cancer. Expert Opin Ther Targets 2007; 11: 1473-1491.

49. Ghiringhelli F, Menard C, Puig PE et al. Metronomic cyclophosphamide regimen selectively depletes $C D 4+C D 25+$ regulatory $T$ cells and restores $T$ and NK effector functions in end stage cancer patients. Cancer Immunol Immunother 2007; 56: 641-648. 